\title{
Discovery of anti-2019-nCoV agents from Chinese patent drugs via docking screening
}

Yong-Ming Yan $^{1, \dagger}$, Xin Shen ${ }^{2, \dagger}$, Yong-Kai Cao ${ }^{1}$, Jiao-Jiao Zhang ${ }^{1}$, Yan Wang, ${ }^{2, *}$, Yong-Xian Cheng ${ }^{1, *}$

${ }^{1}$ School of Pharmaceutical Sciences, Shenzhen University Health Science Center, Shenzhen 518060, P.R. China

${ }^{2}$ Center for Translation Medicine Research and Development, Shenzhen Institute of Advanced Technology, Chinese Academy of Sciences, Shenzhen 518055, P.R. China

* To whom correspondence should be addressed: Tel: 86755-2690 2073; E-mail: ycheng@szu.edu.cn (Y.-X. C.); 86755-2641 7985; E-mail: yan.wang@siat.ac.cn (Y. W.)

${ }^{\dagger}$ These authors contributed equally to this paper.

Running Title: Natural agents against 2019-nCoV by docking screening Keywords: 2019-nCoV, novel coronavirus pneumonia, docking, ACE2, viral main protease 


\section{Abstract}

The 2019 novel coronavirus (2019-nCoV) causes novel coronavirus pneumonia (NCP). Given that approved drug repurposing becomes a common strategy to quickly find antiviral treatments, a collection of FDA-approved drugs can be powerful resources for new anti-NCP indication discoveries. In addition to synthetic compounds, Chinese Patent Drugs (CPD), also play a key role in the treatment of virus related infections diseases in China. Here we compiled major components from 38 CPDs that are commonly used in the respiratory diseases and docked them against two drug targets, ACE2 receptor and viral main protease. According to our docking screening, 10 antiviral components, including hesperidin, saikosaponin, rutin, baicalin, glycyrrhizin, mulberroside A, puerarin, orientin, amygdalin, and ilexgenin A, can directly bind to both host cell target ACE2 receptor and viral target main protease, indicating their potential for 2019-nCoV treatment. 


\section{Introduction}

The 2019 novel coronavirus (2019-nCoV), named as the Wuhan coronavirus [the pneumonia caused by it is now named as novel coronavirus pneumonia (NCP)], is a positive-sense, single-strand RNA coronavirus (1). Up to date, global infections of 2019-nCoV surge past 40,000 (WHO website). Given that drug repurposing is the common strategy to search antiviral treatments, several approved drugs were reported to benefit patients (2). Besides synthetic compounds, natural products, especially Chinese Patent Drug (CPD), also play a key role in the treatment of virus related infections diseases in China. Although the mechanisms of CPDs might be associated with immune regulation, we focus on their antiviral properties. In this study. we compiled major components from 14 out of 38 CPDs that are commonly used in the respiratory diseases and docked them against two drug targets, ACE2 receptor and viral main protease.

Like severe acute respiratory syndrome-related coronavirus (SARS-CoV), the 2019-nCoV attach to host cells through S protein and angiotensin converting enzyme 2 (ACE2) receptor interaction (3). The catalytic inhibitor of ACE2 receptor is likely to induce a conformational change of ACE2, therefore blocking the interaction between $\mathrm{S}$ protein and ACE2 receptor (4). S protein of 2019-nCoV is not currently available but the structure of ACE2 receptor is well-known (5). Thus ACE2 receptor was selected to quickly identify entry inhibitors of 2019-nCoV using marketed CPDs-derived natural products. 
In addition to entry inhibitors, the replication inhibitors are also good strategies for antiviral drug discovery and development (6). Given that 2019-nCoV is a (+)SS RNA virus, its main protease is likely to be required to mediate viral replication and transcription through extensive cleavage of two replicase polyproteins. Therefore inhibition of viral main protease might block virus replication (7). Up to date, Rao et al reported the crystal structure of $\mathrm{M}$ protease of 2019-nCoV (PDB: 6LU7) and several drug repurposing docking screening studies were reported. We herein docked natural product database to main protease to look for antiviral replication agents.

Due to the limited time and lack of the available 2019-nCoV in hand, it is impossible to develop novel compounds against 2019-nCoV by biological screening. We here used docking screening to identify natural products from marketed CPDs that inhibit both virus entry and replication, therefore providing a potential prevention/treatment alternative against 2019-nCoV.

\section{Material and Methods}

The major components of each herb in the selected 14 CPDs were collected as the ligands, and all the ligands were in PDBQT format. The protein model 1R4L was selected as ACE2 receptor docking model while 6LU7 was selected as M protease docking model. Both PDB files of protein models were fetched from Protein Data Bank. The docking screenings were conducted by using AutoDock Vina v.1.0.2. The docking parameters for AutoDock Vina were kept at their default values. The grid box was $25 \AA$ by $25 \AA$ by $25 \AA$, encompassing the catalytic pocket. The binding modes 
were clustered through the root mean square deviation (RMSD) among the Cartesian coordinates of the ligand atoms.

\section{Results and Discussion}

A total of 14 marketed CPDs (bold line in Table 1) containing 63 herbs used for the treatment of respiratory diseases were selected. Totally we docked 52 components (Table 2) and the top 10 hits were summarized in Table 3. All of them provide good binding affinities against both two targets. The key residues for each ligand binding were also summarized in Table 4.

Analysis of the results from Table 3, it was found that the top 10 antiviral components are hesperidin, saikosaponin, rutin, baicalin, glycyrrhizin, mulberroside A, puerarin, orientin, amygdalin, and ilexgenin A, and their binding sites toward 6LU7 and 1R4L are listed in Table 4. A close analysis found that 29 compounds directly bind to ACE2 receptor with high or mild binding affinities (docking score $<-8.5 \mathrm{kcal} / \mathrm{mol}$ ). 14 out of 29 compounds exhibit strong interactions with ACE2 receptor (docking score < $-9.7 \mathrm{kcal} / \mathrm{mol}$ ), these compounds are hesperidin, saikosaponin A (mulberroside A), rutin, baicalin, prim- $O$-glucosylcimifugin, orientin (astragaloside A), ilexgenin A, amygdalin (paeoniflorin), glycyrrhizin, puerarin, and berberine (Table 5). Whereas, in M protease docking screening, 6 phytochemicals, rutin (glycyrrhizin), saikosaponin A, puerarin, hesperidin, baicalin, have been identified as potential $\mathrm{M}$ protease inhibitors (docking score $\leq-8.4 \mathrm{kcal} / \mathrm{mol}$ ), indicating their potential for 2019-nCov. Notably, artemisinin, berberine, rutin, glycyrrhizin, chlorogenic acid, baicalin, cholic 
acid, hyodeoxycholic acid, and puerarin are commercially available with good supply, suggesting a future new drug development is of necessity. In addition, the results of Table 5 in combination of the literature data indicated the natural sources of these active compounds with relatively high content. Basically, around 19 compounds are present in natural sources more than $1 \%(\mathrm{~g} / \mathrm{g})$, which are respectively hesperidin, baicalin, glycyrrhizin, puerarin, amygdalin, paeoniflorin, berberine, arctiin, forsythiaside A, chlorogenic acid, geniposide, magnolol, lobetyolin, pulegone, citrulline, L-menthol, 6-gingerol, rutin (source plant: Potentilla chinensis), and harpagide (source plant: Ajuga pantantha) (Table 5). This information in combination with the docking results suggests that these plants or herbs or their extracts with enriched active compounds might be valuable for fighting against 2019-nCoV. Although the other herbs or CPDs are not found to be active toward 2019-nCoV, this doesn't mean that they are not useful for NCP because only limited compounds in herbs were selected which couldn't exclude more compounds or their analogues in herbs of CPDs are active. In addition, the principles of formulating Chinese herbal prescription include eliminating evil and strengthening the body resistance, therefore, we couldn't exclude that these CPDs do work against NCP via regulating immune system. Further efforts on docking of the structures from the rest 24 CPDs are undergoing. Validation of these docking results is also undergoing.

\section{Acknowledgments}

This study was supported by the National Science Fund for Distinguished Young Scholars (81525026) and National Natural Science Foundation of China (81903875). 


\section{Competing interest statement}

The authors declare no conflict of interest.

\section{References}

1. Huang CL, Wang YM, Li XW, Ren LL, Zhao JP, Hu Y, Zhang L, Fan GH, Xu, JY, Gu, XY, Cheng ZS, Yu, T, Xia JA, Wei A, Wu, WJ, Xie XL, Yin W, Li H, Liu, M, Xiao Y, Gao H, Guo L, Xie JG, Wang GF, Jiang RM, Gao ZC, Jin Q, Wang JW, Cao B. Clinical features of patients infected with 2019 novel coronavirus in Wuhan, China. Lancet, 2020; doi: 10.1016/S0140-6736(20)30183-5.

2. Wang ML, Cao RY, Zhang LK, Yang XL, Liu J. Xu MY, Shi ZL, Hu ZH, Zhong W, Xiao GF. Remdesivir and chloroquine effectively inhibit the recently emerged novel coronavirus (2019-nCoV) in vitro. Cell Res., 2020; doi: 10.1038/s41422-020-0282-0.

3. Wan YS, Shang J, Graham R, Baric RS, Li F. Receptor recognition by novel coronavirus from Wuhan: An analysis based on decade-long structural studies of SARS. J. Virol., 2020; doi: 10.1128/JVI.00127-20.

4. Du LY, He YX, Zhou YS, Liu SW, Zheng BJ, Jiang SB. The spike protein of SARS-CoV -a target for vaccine and therapeutic development. Nat. Rev. Microbiol., 2009; 7(3): 226-236.

5. Towler P, Staker B, Prasad SG, Menon S, Tang J, Parsons T, Ryan D, Fisher M, Williams D, Dales NA, Patane MA, Pantoliano MW. ACE2 X-ray structures reveal a large hinge-bending motion important for inhibitor binding and catalysis. J. Biol. 
Chem., 2004; 279(17):17996-8007.

6. Clercq ED. Strategies in the design of antiviral drugs. Nat. Rev. Drug Discov., 2002; 1: $13-25$.

7. Wit ED, van Doremalen NV, Falzarano D, Munster VJ. SARS and MERS: recent insights into emerging coronaviruses. Nat. Rev. Microbiol., 2016; 14: 523-534. 
Table 1. Commercial names of 38 Chinese patent drugs (CPDs)

\begin{tabular}{|c|c|c|c|}
\hline No. & CPDs & No. & CPDs \\
\hline 1 & Fengre Ganmao Granules & 20 & Kangbingdu Capsules \\
\hline 2 & Xiaochaihu Granules & 21 & Fufang Banlangen Granules \\
\hline 3 & Qingkailing Capsules & 22 & $\begin{array}{l}\text { Ganmao Shufeng } \\
\text { Capsules/Granules }\end{array}$ \\
\hline 4 & Jinlianhua Capsules & 23 & Ganmao Qingre Granules \\
\hline 5 & Zhongganling Capsules & 24 & Fufang Jinyinhua Granules \\
\hline 6 & $\begin{array}{l}\text { Lianhua Qingwen } \\
\text { Capsules/Granules }\end{array}$ & 25 & Yinqiao Jiedu Pills/Granules \\
\hline 7 & Lanqin Oral Solution & 26 & Vitamin C Yinqiao Tablets \\
\hline 8 & Qingwen Jiedu Tablets & 27 & $\begin{array}{l}\text { Fufang Yinqiao Anfen } \\
\text { Capsules }\end{array}$ \\
\hline 9 & Fangfeng Tongsheng Pills & 28 & Xiasangju Granules \\
\hline 10 & $\begin{array}{l}\text { Shuanghuanglian Oral } \\
\text { Solution }\end{array}$ & 29 & $\begin{array}{l}\text { Vitamin C Effervescent } \\
\text { Tablets }\end{array}$ \\
\hline 11 & $\begin{array}{l}\text { Huoxiang Zhengqi Oral } \\
\text { Solution }\end{array}$ & 30 & Xiaoer Ganmao Granules \\
\hline 12 & Huoxiang Zhengqi Capsules & 31 & Banlangen Granules \\
\hline 13 & Maxing Zhike Syrup & 32 & Qingkailing Oral Solution \\
\hline 14 & Choulingdan Oral Solution & 33 & Yinqiao Jiedu Granules \\
\hline 15 & Erding Capsules & 34 & $\begin{array}{l}\text { Fufang Yinqiao Anfen } \\
\text { Vitamin C Tablets }\end{array}$ \\
\hline 16 & Zhiganjia Granules & 35 & Ganmao Soft Capsules \\
\hline 17 & Kanggan Granules & 36 & Fenghan Ganmao Granules \\
\hline 18 & Kangbingdu Granules & 37 & Qiangli Pipa Syrup \\
\hline 19 & Kangbingdu Oral Emulsion & 38 & Fufang Anwanan Tablets \\
\hline
\end{tabular}


Table 2. Appendix total docking ranking

\begin{tabular}{|c|c|c|c|}
\hline Ligand & 6LU7 & 1R4L & Sum \\
\hline Hesperidin & -8.5 & -11.4 & -19.9 \\
\hline Saikosaponin A & -8.8 & -11 & -19.8 \\
\hline Rutin & -8.9 & -10.7 & -19.6 \\
\hline Baicalin & -8.4 & -10.5 & -18.9 \\
\hline Glycyrrhizin & -8.9 & -9.9 & -18.8 \\
\hline Mulberroside A & -7.7 & -11 & -18.7 \\
\hline Puerarin & -8.6 & -9.8 & -18.4 \\
\hline Orientin & -8.1 & -10.2 & -18.3 \\
\hline Amygdalin & -8.1 & -10 & -18.1 \\
\hline Ilexgenin A & -7.9 & -10.1 & -18 \\
\hline Prim-O-glucosylcimifugin & -7.6 & -10.4 & -18 \\
\hline Astragaloside A & -7.6 & -10.2 & -17.8 \\
\hline Paeoniflorin & -7.7 & -10 & -17.7 \\
\hline Nodakenin & -7.9 & -9.6 & -17.5 \\
\hline Swertiajaponin & -8 & -9.4 & -17.4 \\
\hline Berberine & -7.5 & -9.7 & -17.2 \\
\hline Arctiin & -7.3 & -9.5 & -16.8 \\
\hline Forsythiaside A & -7.6 & -9.1 & -16.7 \\
\hline Radix isatidis A & -7.6 & -9.1 & -16.7 \\
\hline Indirubin & -7.3 & -9.3 & -16.6 \\
\hline Artemisinin & -7.3 & -9.1 & -16.4 \\
\hline Emodin & -7.2 & -9.2 & -16.4 \\
\hline Cholic acid & -7 & -9.3 & -16.3 \\
\hline Hyodeoxycholic acid & -7 & -9.3 & -16.3 \\
\hline Xanthiside & -7.3 & -8.9 & -16.2 \\
\hline Chlorogenic acid & -7.3 & -8.8 & -16.1 \\
\hline
\end{tabular}




\begin{tabular}{|c|c|c|c|}
\hline Verbenalin & -7.4 & -8.7 & -16.1 \\
\hline Poricoic acid A & -6.9 & -9.2 & -16.1 \\
\hline Notopterol & -7 & -8.4 & -15.4 \\
\hline Harpagide & -7 & -8.3 & -15.3 \\
\hline Imperatorin & -7.1 & -8.2 & -15.3 \\
\hline Geniposide & -6.7 & -8.5 & -15.2 \\
\hline Salidroside & -6.9 & -7.9 & -14.8 \\
\hline Atractylenolide I & -6.3 & -8.2 & -14.5 \\
\hline Magnolol & -6.4 & -7.9 & -14.3 \\
\hline Lobetyolin & -6.4 & -7.7 & -14.1 \\
\hline Pterodontic acid & -6 & -7.7 & -13.7 \\
\hline Platycodin D & -7.5 & -5.5 & -13 \\
\hline Dhelwangin & -5.2 & -7.1 & -12.3 \\
\hline Ferulic acid & -5.4 & -6.5 & -11.9 \\
\hline 6-Gingerol & -4.8 & -6.6 & -11.4 \\
\hline Atractylodin & -4.9 & -6.3 & -11.2 \\
\hline Ephedrine & -5.1 & -6.1 & -11.2 \\
\hline Pulegone & -4.9 & -6.2 & -11.1 \\
\hline Citrulline & -4.9 & -5.8 & -10.7 \\
\hline Linolenic acid & -4.6 & -6.1 & -10.7 \\
\hline L-Menthol & -4.7 & -5.7 & -10.4 \\
\hline Arecoline & -4.6 & -5.4 & -10 \\
\hline Glutamic acid & -4.5 & -5.3 & -9.8 \\
\hline Tetramethyl pyrazine & -4.5 & -5.1 & -9.6 \\
\hline Succinic acid & -4.4 & -4.9 & -9.3 \\
\hline Decanoy acetaldehyde & -3.9 & -4.9 & -8.8 \\
\hline
\end{tabular}


Table 3. Natural products from CPDs docking results

\begin{tabular}{lccc}
\hline \multirow{2}{*}{ Ligand } & \multicolumn{3}{c}{ Binding affinity (kcal/mol) } \\
\cline { 2 - 4 } & 6LU7 & 1R4L & Sum \\
\hline Hesperidin & -8.5 & -11.4 & -19.9 \\
Saikosaponin & -8.8 & -11 & -19.8 \\
Rutin & -8.9 & -10.7 & -19.6 \\
Baicalin & -8.4 & -10.5 & -18.9 \\
Glycyrrhizin & -8.9 & -9.9 & -18.8 \\
Mulberroside A & -7.7 & -11 & -18.7 \\
Puerarin & -8.6 & -9.8 & -18.4 \\
Orientin & -8.1 & -10.2 & -18.3 \\
Amygdalin & -8.1 & -10 & -18.1 \\
Ilexgenin A & -7.9 & -10.1 & -18 \\
\hline
\end{tabular}


Table 4. Key residues for potential inhibitors binding

\begin{tabular}{|c|c|c|}
\hline \multirow{2}{*}{ Ligand } & \multicolumn{2}{|c|}{ Key residues } \\
\hline & 6LU7 & 1R4L \\
\hline Hesperidin & $\begin{array}{l}\text { Gly143, Ser144, Cys145, } \\
\text { Glu166 }\end{array}$ & $\begin{array}{l}\text { Cy3344, His345, Asp368, Arg514, } \\
\text { Tyr515, Arg518 }\end{array}$ \\
\hline Saikosaponin & $\begin{array}{l}\text { His41, Glu166, Arg188, } \\
\text { Gln189, Thr190, Gln192 }\end{array}$ & $\begin{array}{l}\text { Ala348, Glu402, Arg514, Tyr515, } \\
\text { Arg518 }\end{array}$ \\
\hline Rutin & $\begin{array}{l}\text { His163, Phe140, Glu166, } \\
\text { Arg188 }\end{array}$ & $\begin{array}{l}\text { Asn149, Arg273, His345, Thr445, } \\
\text { His505, Tyr515 }\end{array}$ \\
\hline Baicalin & $\begin{array}{l}\text { Thr25, Thr26, Leu141, } \\
\text { Gly143, Ser144, Cys145 }\end{array}$ & $\begin{array}{l}\text { His345, Lys363, Thr371, His505, } \\
\text { Arg518 }\end{array}$ \\
\hline Glycyrrhizin & $\begin{array}{l}\text { Phe140, His163, His164, } \\
\text { Arg188 }\end{array}$ & $\begin{array}{l}\text { Arg273, His345, Thr365, Thr371, } \\
\text { Tyr515, Arg518 }\end{array}$ \\
\hline $\begin{array}{l}\text { Mulberroside } \\
\text { A }\end{array}$ & $\begin{array}{l}\text { Thr24, Thr26, Gly143, } \\
\text { Ser144, Cys145, Gln189 }\end{array}$ & $\begin{array}{l}\text { Asn149, Arg273, Lys363, Asp367, } \\
\text { Asp368, Tyr515, Arg518 }\end{array}$ \\
\hline Puerarin & $\begin{array}{l}\text { Thr26, His41, Leu141, } \\
\text { Ser144, His163, Glu166 }\end{array}$ & Asn149, Asp367, Glu402, Tyr515 \\
\hline Orientin & $\begin{array}{l}\text { Phe140, Glu166, Gln189, } \\
\text { Thr190 }\end{array}$ & $\begin{array}{l}\text { Arg273, Thr371, Glu406, Tyr515, } \\
\text { Arg518 }\end{array}$ \\
\hline Amygdalin & $\begin{array}{l}\text { Leu141, Gly143, Ser144, } \\
\text { Glu166, Arg188 }\end{array}$ & Asn149, Pro346, Lys363, Arg518 \\
\hline Ilexgenin A & Thr24, Leu141, Gly143 & His345, Lys363, Thr371 \\
\hline
\end{tabular}


Table 5. The structure, natural source and content of active components, and weight ratio of a herb in Chinese patent drugs

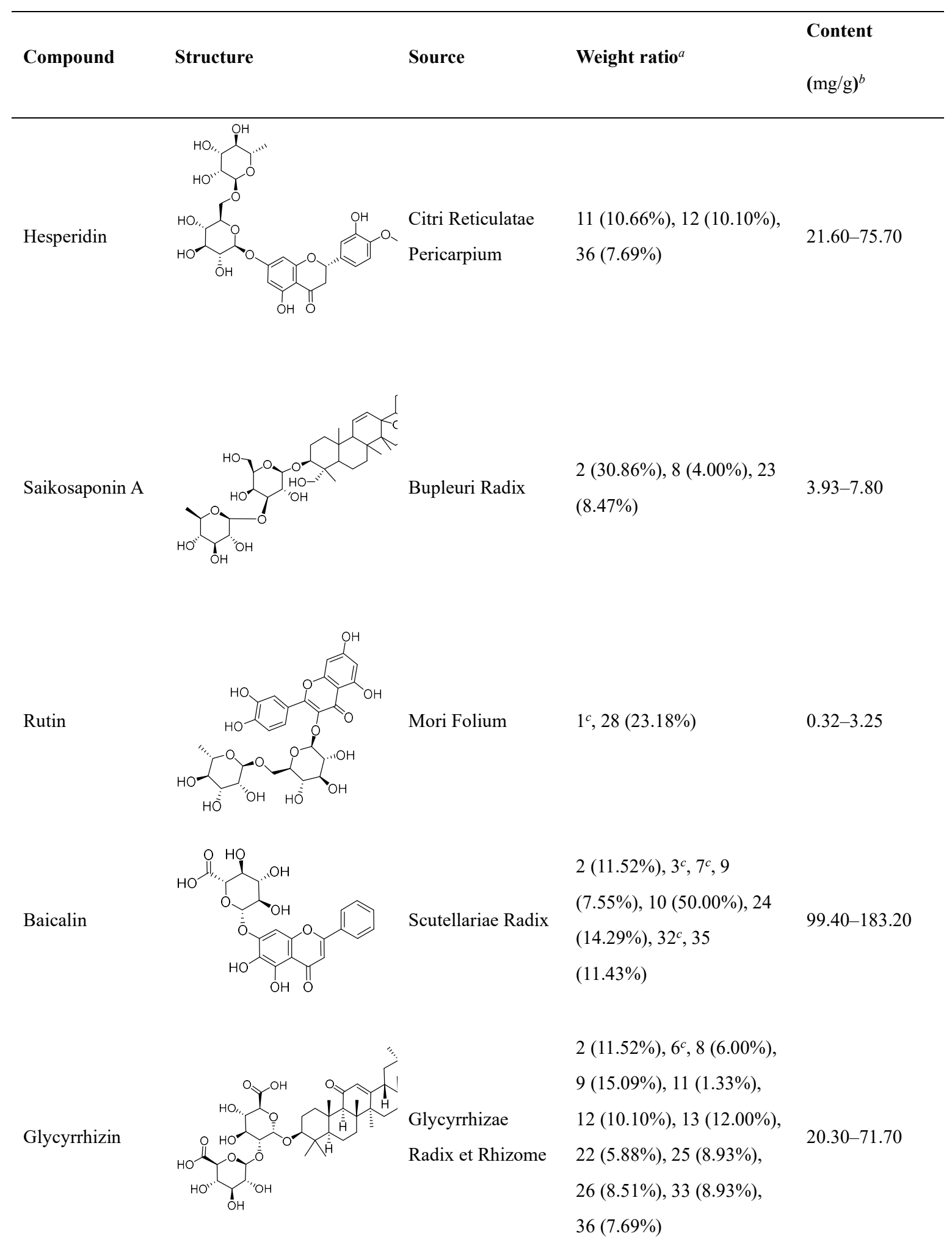


lberroside A<smiles>CCOc1ccc(/C=C/c2cc(O)c(OC3OC(CO)[C@@H](O)[C@H](O)C3O)c(O)c2)c(O)c1</smiles>

Puerarin<smiles>O=c1c(-c2ccc(O)cc2)coc2c(C3O[C@H](CO)[C@@H](O)[C@H](O)C3O)c(O)ccc12</smiles>
Puerariae Lobatae $5^{c}, 8$ (8.00\%), 23 (8.47\%), 35 (8.57\%), 36 (11.54\%)

Orientin<smiles>O=c1cc(-c2ccc(O)c(O)c2)oc2c(O)c(O)cc(O)c12</smiles>

Amygdalin<smiles>N#CC1OC(OCC2OC(CO)C(O)C(O)C2O)C(O)C(O)C1O</smiles>

\section{Armeniacae}

Semen Amarum

$1^{c}, 6^{c}, 13(16.00 \%), 22$

(8.82\%), 23 (6.78\%), 35

$36.7-45.8$

(11.43\%), $36(11.54 \%)$

Ilicis Pubescentis

Radix et Caulis

$5^{c}$

4.1-15.6

Ilexgenin A

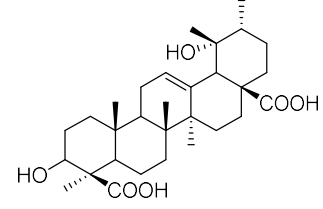

Saposhnikoviae

Prim- $O$-glucosyl cimifugin

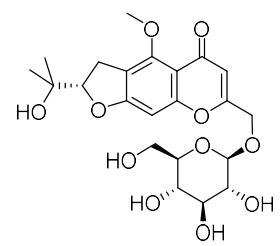

Radix

8 (4.00\%), 9 (3.77\%), 22

(8.82\%), 23 (8.47\%), 35

(5.71\%), $36(11.54 \%)$

Astragali Radix $\quad 8(8.00 \%)$

$0.26-2.13$

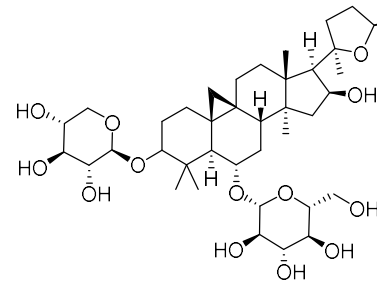

Paeoniae Radix

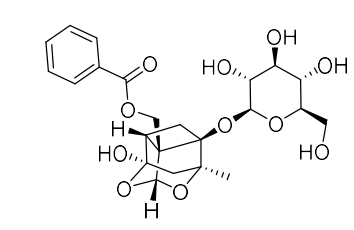

Paeoniae Radix
Paeoniflorin
Rubra

Alba
Paeoniae Radix Rubra : 8

(4.00\%), 17 (42.87\%)

Paeoniae Radix Alba : 9

$20.00-25.00$

(3.77\%), $22(14.70 \%)$ 
Nodakenin<smiles></smiles>

Notopterygii

Rhizoma Et Radix

$5^{c}, 8(6.00 \%), 16^{c}, 35$

$(5.71 \%)$

8 (8.00\%), 25 (7.14\%), 26,

Lophatheri Herba

Swertiajaponin<smiles>CCc1cc2oc(-c3ccc(O)c(O)c3)cc(=O)c2c(O)c1C1OC(CO)[C@@H](C)[C@H](O)C1O</smiles>

Phellodendri

Berberine<smiles>COc1ccc2cc3c(cc2c1OC)[N+]1=C(CC1)c1cc2c(cc1-2)OCO3</smiles>

Chinensis

Cortex

Arctiin<smiles>COc1ccc(CC2CCOC2=O)cc1CC1CC(CO)[C@@H](O)C(O)C1Oc1ccc(OC)c(OC)c1</smiles><smiles>C=CC(=C)CCOC1OC(OC2O[C@@H](C)[C@H](C)[C@@H](C)O2)C(O)C(O)C1O</smiles>

Forsythiaside A<smiles>C/C=C(/C)C(=O)COC1c2c(ccc3ccc(=O)[nH]c23)O[C@H]1C(C)(C)OC(C)=O</smiles>

Indirubin

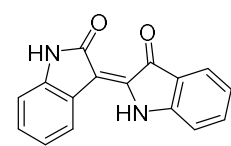

Artemisinin

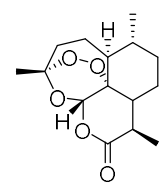<smiles>Cc1cc(O)c2c(c1)C(=O)c1cc(O)cc(O)c1C2=O</smiles>

Arctii Fructus

Forsythiae Fructus

Isatidis Radix

Isatidis Folium

$8^{c}, 21(60.00 \%), 30$

$(15.15 \%)$

$0.02-4.15$

Artemisiae

Annuae Herba

$1^{c}, 8(8.00 \%), 25$

$47.5-73.3$

$(10.71 \%)$

$1^{c}, 6^{c}, 8(6.00 \%), 9$

(3.77\%), 10 (25.00\%), 19

$20^{c}, 24$ (42.86\%), 25

$146.2-172.1$

$27^{c}, 30(9.09 \%), 33$

(17.86\%), $34^{c}$

$1^{c}, 3^{c}, 5^{c}, 6^{c}, 7^{c}, 15^{c}, 16^{c}$,

$18^{c}, 19^{c}, 20^{c}, 21(40.00 \%)$, $30(9.09 \%), 31(100 \%)$,

Unknown

$32^{c}$

$5^{c}$

$1.91-5.19$

Rhei Radix Et

Rhizoma $6^{c}, 9(3.77 \%)$

$0.29-0.66$
(10.71\%), 26 (10.21\%), 33

(17.86\%), $26(17.01 \%)$

Emodin 
Cholic acid

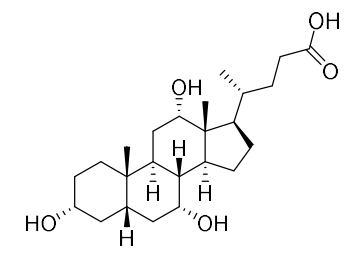

Cholic acid

$3^{c}, 32^{c}$

Hyodeoxycholic acid<smiles>C=C1CSC2=C(N1)C(=O)C=C(CC1OC(CO)[C@H](C)C(O)C1O)C2(C)C</smiles>

Hyodeoxycholic

acid

Massa Medicata

Fermentata

$1^{c}$

$3^{c}, 32^{c}$

Lonicerae Japonicae Flos:

$3^{c}, 6^{c}, 10$ (25.00\%), 17

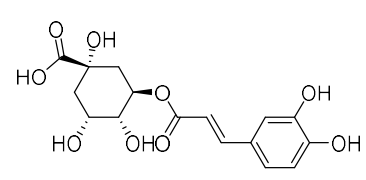

Lonicerae

Japonicae Flos

Chrysanthemi

Flos

Verbenae Herba $\quad 5^{c}$

(42.87\%), 24 (42.86\%), 25

(17.86\%), 27c $, 32^{c}, 33$

$(17.86 \%), 34^{c}$

22.30-31.60;

$2.38-7.20$

Chrysanthemi Flos: 28

(10.59\%), 30 (9.09\%)<smiles>COC(=O)C1=COC2C(OC3OC(CO)C(O)C(O)C3O)C3C(=O)CC[C@H](C)C3C12</smiles>

$1.52-3.35$

$11(16.00 \%), 12(5.05 \%)$

$0.24-0.40$

Poricoic acid A

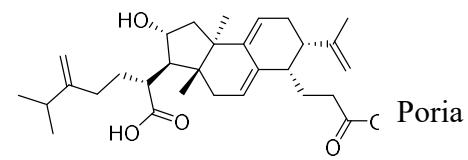

Notopterygii

Notopterol

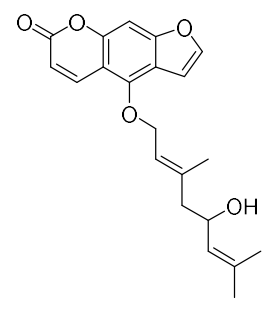

Rhizoma Et Radix

$5^{c}, 16,35(5.71 \%)$

$3.50-15.00$ 
Harpagide

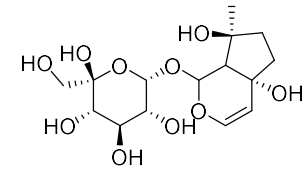

Imperatorin<smiles>CC(C)=CCCc1c2occc2cc2ccc(=O)oc12</smiles>

Geniposide<smiles>CCOC(=O)C1=CCC(OC2OC(CO)C(O)C(O)C2O)C2CC=C(CO)C12</smiles>

Salidroside<smiles>CCC1OC(OCCc2ccc(O)cc2)[C@H](O)[C@@H](O)[C@H]1O</smiles>

Atractylenolide I<smiles></smiles>

Magnolol<smiles>C=CCc1ccc(O)c(-c2cc(CC=C)ccc2O)c1</smiles><smiles>C/C=C/C#CC#CC(O)C(/C=C/CCCO)OC1OC(CO)[C@H](C)C(O)[C@H]1O</smiles>

Laggerae Herba<smiles>C=C(C(=O)O)C1C=C2C(C)CCCC2(C)CC1</smiles>

Platycodin D

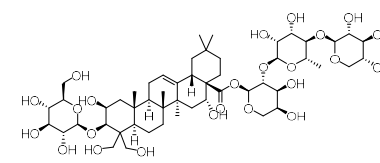<smiles>Cc1cc(O)c(C(=O)CCC(C)C)c(=O)o1</smiles>

Herba
Codonopsis Radix

Lobeliae

Chinensis Herba

Platycodonis

Radix

Pogostemonis

Scrophulariae

Radix

$8(8.00 \%)$

$3.59-4.86$

8 (4.00\%), 11 (16.00\%),

$12(5.05 \%), 18^{c}, 23$

(5.08\%), 35 (5.71\%), 36

$(7.69 \%)$

Rhodiolae

Crenulatae Radix

$6^{c}$

7.83-11.09

Et Rhizoma

Atractylodis

Macrocephalae

$9(1.89 \%), 12(10.10 \%)$

$1.93-2.54$

Rhizoma

Magnoliae

Officinalis Cortex

$11(10.66 \%), 12(10.10 \%)$

$9.50-67.80$

Codonopsis Radix: 2

(11.52\%)

$29.50-59.40$

Lobeliae Chinensis Herba:

$15^{c}$

$14(100 \%)$

Unknown

$8(6.00 \%), 9(7.55 \%), 12$

(10.10\%), 22 (5.88\%), 23

(5.08\%), 25 (10.71\%), 26

(10.21\%), 33 (10.71\%), 35

$1.85-4.06$

(5.71\%), 36 (7.69\%), 37

$(3.72 \%)$

$6^{c}, 11(5.33 \%), 12$

$(15.15 \%), 19^{c}, 20^{c}, 30$

$1.35-5.71$

$(9.09 \%)$ 
Ferulic acid<smiles>COc1cc(/C=C/C(=O)O)ccc1O</smiles>

6-Gingerol<smiles>CCCCCC(O)CC(=O)CCc1ccc(O)c(OC)c1</smiles>

Atractylodin

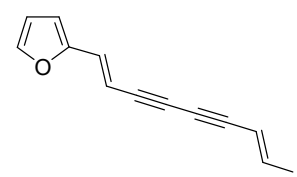

Ephedrine

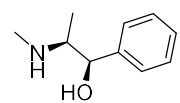

Pulegone

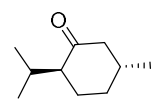

Citrulline<smiles>NC(=O)NCCC[C@H](N)C(=O)O</smiles>

Linolenic acid

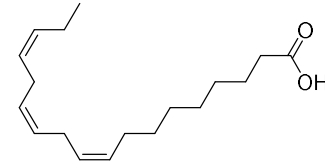

L-Menthol<smiles>CC1CCC(C(C)C(C)C)C(O)C1</smiles>

Arecoline<smiles>COC(=O)C1=CCCN(C)C1</smiles>

Glutamic acid
Phragmitis

Rhizoma

Angelicae

Sinensis Radix

Zingiberis

Rhizoma Recens

$2(11.52 \%), 12(1.51 \%)$,

$22(5.88 \%), 36(7.69 \%)$

9.96-28.64

Atractylodis

Rhizoma

$11(10.66 \%)$

$3.98-10.96$

13 (24.0\%), 22 (5.88\%),

Ephedrae Herba

35 (11.43\%), 36 (7.69\%),

$4.80-10.20$

$9(3.77 \%), 6^{c}$

$1^{c}, 9(1.89 \%), 23$

Schizonepetae

Spica

(16.95\%), 25 (7.14\%), 26

Volatile oils

(6.80\%), 27,33 (7.14\%),

294.27-754.02

$34,35(8.57 \%)$

Trichosanthis

Radix

$8(8.00 \%)$

$20.20-60.20$

11 (2.67\%), 12 (5.05\%),

Perillae Folium

22 (5.88\%), 23 (5.08\%),

$36(11.54 \%)$

$9(3.77 \%), 16^{c}, 23$

Menthae

(5.08\%), 25 (10.71\%), 26

Haplocalycis

(4.73\%), 27c, $30(6.06 \%)$

25.76-226.10

Herba

$33(10.71 \%), 34^{c}, 35^{c}, 1^{c}$,

$37(3.72 \%)$

Arecae

Pericarpium

$11(16.00 \%), 12(5.05 \%)$

$1.92-3.80$
0.09-0.68 $32^{c}$<smiles>N[C@@H](CCC(=O)O)C(=O)O</smiles>

Bubali Cornu 


\begin{tabular}{|c|c|c|c|}
\hline $\begin{array}{l}\text { Tetramethyl } \\
\text { pyrazine }\end{array}$ & $\begin{array}{l}\text { Chuanxiong } \\
\text { Rhizoma }\end{array}$ & $\begin{array}{l}35(5.71 \%), 8(4.00 \%), 9 \\
(3.77 \%)\end{array}$ & $0.15-0.24$ \\
\hline Succinic acid & Pinelliae Rhizoma & $\begin{array}{l}2(11.52 \%), 11(10.66 \%), \\
12(10.10 \%)\end{array}$ & $3.24-4.43$ \\
\hline $\begin{array}{l}\text { Decanoy } \\
\text { acetaldehyde }\end{array}$ & $\begin{array}{l}\text { Houttuyniae } \\
\text { Herba }\end{array}$ & $6^{c}, 18^{c}$ & $\begin{array}{l}\text { Volatile oils } \\
7.2 \%\end{array}$ \\
\hline
\end{tabular}

${ }^{a}$ The number of Chinese patent drugs is same as that in table 1 .

${ }^{b}$ Data source: China National Knowledge Infrastructure (CNKI).

${ }^{c}$ Unknown. 